\title{
Moving eyes and moving thought: On the spatial compatibility between eye movements and cognition
}

\author{
LAura E. Thomas AND Alejandro Lleras \\ University of Illinois at Urbana-Champaign, Urbana, Illinois
}

\begin{abstract}
Grant and Spivey (2003) proposed that eye movement trajectories can influence spatial reasoning by way of an implicit eye-movement-to-cognition link. We tested this proposal and investigated the nature of this link by continuously monitoring eye movements and asking participants to perform a problem-solving task under free-viewing conditions while occasionally guiding their eye movements (via an unrelated tracking task), either in a pattern related to the problem's solution or in unrelated patterns. Although participants reported that they were not aware of any relationship between the tracking task and the problem, those who moved their eyes in a pattern related to the problem's solution were the most successful problem solvers. Our results support the existence of an implicit compatibility between spatial cognition and the eye movement patterns that people use to examine a scene.
\end{abstract}

For over 30 years, researchers have used eyetracking techniques to gain insight into cognitive processing. The examination of eye fixations and eye movements during diagram-based problem solving has given us a better understanding of problem-solving strategies in a wide variety of tasks such as mental rotation, insight problem solving, and inference making (see, e.g., Just \& Carpenter, 1985; Knoblich, Ohlsson, \& Raney, 2001; Lenhart, 1983). Although researchers have long investigated how cognitive processes influence eye movements, only recently have they begun to look into the reciprocal relationship and ask how eye movements might influence cognitive processes.

A recent study by Grant and Spivey (2003) began to address the question of whether eye movements can direct cognitive processing during a problem-solving task using a classic insight problem ${ }^{1}$ : Karl Duncker's (1945) radiation problem. Figure $1 \mathrm{~A}$ presents a diagram of this problem. In their study, Grant and Spivey showed participants a similar diagram and gave them the following instructions (diagram and instructions adapted by Grant \& Spivey, 2003, from Duncker, 1945):

Given a human being with an inoperable stomach tumor, and lasers which destroy organic tissue at sufficient intensity, how can one cure the person with these lasers and, at the same time, avoid harming the healthy tissue that surrounds the tumor?

The correct solution to this problem entails firing multiple low-intensity lasers from different locations around the tumor so that they converge at the tumor. Although each individual laser is too weak to damage the healthy tissue surrounding the tumor, the combined intensity of multiple lasers that meet at the tumor is enough to destroy it. In this problem, the relevant areas are the inner black oval representing the tumor, the outer black oval representing the skin and the healthy tissue it encompasses, and the white area beyond the skin representing the outside area from which the multiple lasers must fire.

In their first experiment, Grant and Spivey (2003) recorded the eye movements of participants attempting to solve the radiation problem. They found that participants who successfully solved the problem within 10 min without hints spent more time looking at the skin area than did participants unable to solve the problem without hints. On the basis of this finding, Grant and Spivey concluded that the skin area was critical for inferring the problem's solution. In a second experiment, in which eye movements were not recorded, they attempted to direct a group of participants' attention to this critical area by presenting them with a problem diagram in which the skin pulsed. Participants who viewed a skin-pulsing diagram had a higher rate of problem-solving success than did those who viewed a static diagram, or those who viewed a diagram in which a noncritical area, the tumor, pulsed.

What was special about the skin area in Grant and Spivey's (2003) experiments? The researchers suggested that increasing the time that participants spent viewing the skin area also increased triangular in-and-out eye movement patterns, in which participants looked to the outside area, moved their eyes across the skin and into the tumor area, and then moved back out again to another outside area. This in-and-out pattern of eye movements actually 


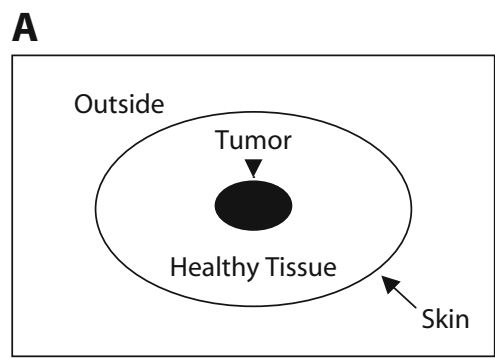

B
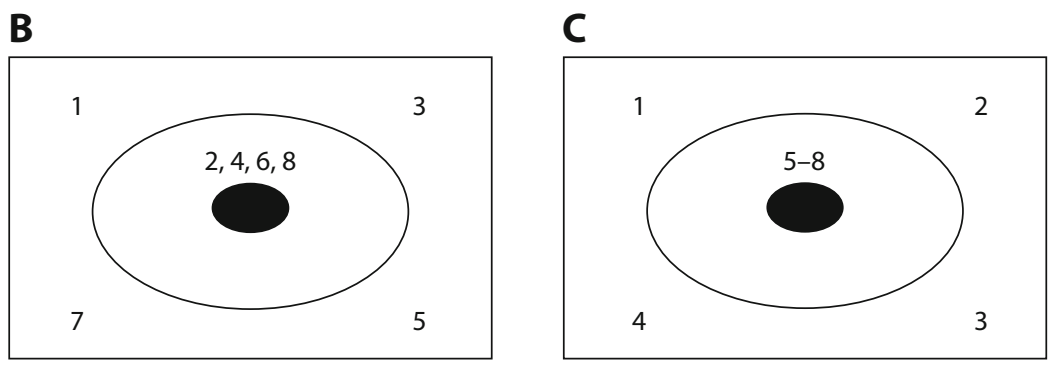

D

E
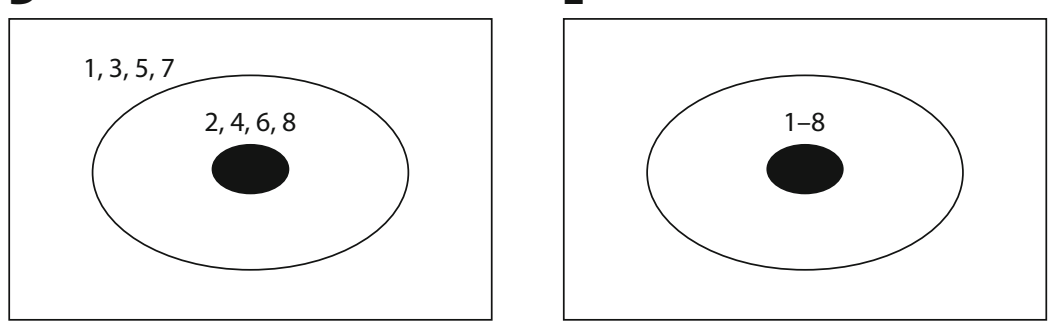

Figure 1. Diagram of Duncker's (1945) radiation problem. Panel B shows the letter/ digit sequence locations for the embodied-solution group, panel $\mathrm{C}$ shows the sequence for the areas-of-interest group, panel $D$ shows the sequence for the repeated-skincrossing group, and panel $\mathbf{E}$ shows the sequence for the tumor-fixation group.

embodies the solution to the radiation problem; the eyes draw a path that reflects multiple lasers converging from different outer areas at the tumor. Grant and Spivey found that successful problem solvers in their first experiment made significantly more skin-crossing in-and-out saccades than did unsuccessful problem solvers. They suggested that these skin-crossing saccades acted as an embodied physical mechanism that initiated a perceptual simulation (Barsalou, 1999) of multiple lasers fired from different points outside the diagram. They concluded that eye movements guided cognitive processing in the radiation problem task; these results provide an example of embodied cognition (e.g., Wilson, 2002).

Grant and Spivey's (2003) work suggests that cognitive processing and eye movement patterns are linked. It also raises interesting questions about the nature of the relationship between eye movements and problem solving. By devising a task that manipulated participants' eye movements as they viewed a problem diagram, we investigated the hypothesis that eye movements can act as an embodied mechanism that guides cognitive processing. Whereas Grant and Spivey found that successful problem solvers tended to move their eyes in a pattern that embodied the problem's solution, we investigated whether we could turn participants into successful problem solvers by forcing them to move their eyes in a pattern that embodied the problem's solution. To do so, we occasionally guided the eye movements of participants with a tracking task while they attempted to solve Duncker's (1945) radiation problem. The tracking task was unrelated to the radiation problem and consisted of identifying a digit among letters presented at different locations within the problem diagram. Participants were assigned to one of four different groups. These groups differed solely in the pattern in which eye movements were guided during the tracking task.

Participants in the embodied-solution group made many skin-crossing saccades to several locations during the tracking task, emphasizing the in-and-out pattern that embodies multiple lasers converging on the tumor. Participants in the areas-of-interest group made eye movements to the same outer locations as did participants in the embodied solution group, but without making a large number of skin-crossing saccades. Like participants in the embodied-solution group, participants in the repeatedskin-crossing group made many skin-crossing saccades, but all of these saccades were made between the same two points, deemphasizing the solution pattern of multiple lasers converging on the tumor. Participants in the tumor-fixation group kept their eyes fixated on the tumor during the tracking task. Differences in the problem- 
solving success rates between these groups should help us to determine the effectiveness of eye movement patterns in directing cognitive processing. If skin-crossing saccades that embody the solution to Duncker's (1945) radiation problem are the key to helping participants arrive at the necessary insight, we should observe a higher success rate in the embodied-solution group than in any of the other groups. If, however, simply directing the eyes to critical outer regions of the diagram is a sufficient aid to insight, success rates of both the embodied-cognition and areas-of-interest groups should be higher than those of the other groups. If skin-crossing saccades are an aid to insight (regardless of the extent to which they embody the solution), participants in both the embodied-solution and repeated-skin-crossing groups should have the highest success rates. Finally, if guiding the eyes has no influence on participants' cognition, we would expect all groups to have similar rates of problem-solving success.

\section{METHOD}

\section{Participants}

Ninety-nine undergraduate students participated in one experimental session approximately $30 \mathrm{~min}$ long. Participants who failed to reach $80 \%$ accuracy on the tracking task were excluded from analyses. Also excluded from the analyses were data from participants who reported being aware of a link between the tracking and problem-solving tasks (see Results section, below, for details). Table 1 shows the total number of participants in each condition as well as the number dropped from each condition, leaving us with a total of 18 acceptable participants each in the embodied-solution, areas-of-interest, and tumor-fixation groups, and 16 acceptable participants in the repeated-skin-crossing group. Each participant received $\$ 8$ for participating.

We ran the experiment in two parts. In Part 1, participants were randomly assigned to one of three experimental groups-the embodied-solution, areas-of-interest, or tumor-fixation group. In Part 2, run at a later time, all participants were assigned to the repeated-skin-crossing group. ${ }^{2}$

\section{Stimuli and Apparatus}

The stimuli consisted of the diagram in Figure 1, which constitutes the problem space for Duncker's (1945) radiation problem, and a random selection of letters and digits that were presented within this space. The display background was white and all stimuli were black.

Stimuli were presented on a 21-in. monitor with resolution of $1,024 \times 768$ pixels. An EyeLink II video-based eyetracker (SR Research Ltd., Mississauga, Ontario, Canada) with a temporal resolution of $500 \mathrm{~Hz}$, spatial resolution of $0.1^{\circ}$, and pupil-size resolution of $0.1 \%$ of pupil diameter recorded eye movements. ${ }^{3}$ It classified an eye movement as a saccade when its distance exceeded $0.2^{\circ}$ and its velocity reached $30^{\circ} / \mathrm{sec}$, or when its distance exceeded $0.2^{\circ}$ and its acceleration reached $9,500^{\circ} / \mathrm{sec}$.

During the experiment, participants sat $58 \mathrm{~cm}$ from the display monitor, their heads stabilized on a chinrest. At this viewing distance, the outer oval within the problem space subtended approximately $20.3^{\circ}$ of visual angle horizontally and $14.0^{\circ}$ vertically. Individual letters and digits subtended approximately $0.3^{\circ}$ horizontally and $0.5^{\circ}$ vertically. The drift correction dot presented at the start of each trial subtended $0.7^{\circ}$. Participants made their responses via keys on a game pad interfaced with the EyeLink software.

\section{Procedure}

Participants were first questioned on their familiarity with Duncker's (1945) radiation problem. All participants were naive to the problem's solution. Participants were fitted with the eyetracker and a calibration procedure was run. They then saw a screen that displayed the problem diagram and written instructions detailing the radiation problem and the letter/digit tracking task, which an assistant read aloud to participants. Following the instructions, a participant pressed a game pad key to begin the experiment.

\section{Free-Viewing Period}

The experiment was divided into twenty 30 -sec intervals. Each interval consisted of a 26-sec free-viewing period and a 4-sec digittracking task. At the start of each 30-sec interval, a brief drift correction procedure was performed in which participants fixated a dot in the center of the display and pressed a game pad key with the left thumb. Following successful drift correction, the problem diagram appeared alone on screen for $25 \mathrm{sec}$. During this 25 -sec period, participants were under no instruction about how to move their eyes or where to fixate within the display.

\section{Tracking Task}

Following the free-viewing period, a random string of 7 letters and 1 digit was presented at various onscreen locations, at a rate of $500 \mathrm{msec} / \mathrm{item}$. The digit could appear at any position within the eight-item sequence. As soon as participants detected the digit, they pressed the game pad key under the right index finger. Because of the small size of the digit and letter stimuli, participants had to foveate each item in order to successfully perform this task. Participants' reaction times (RTs) to detect the digit were measured. Eye movements were recorded during the entire length of the experiment.

The four experimental groups differed on the basis of the pattern in which letters and digits appeared during the tracking task. Figure 1 illustrates the differences between groups. For each group, the sequence of locations at which the tracking items appeared was the same for every trial.

The tracking task for the embodied-solution group emphasized triangular in-and-out eye movements that crossed from the outside area, over the skin, to the tumor on the problem diagram, and then back out to a different location of the outside area. The letter/digit sequence was presented, in order, over the following locations,

Table 1

Number of Participants, Tracking Task Reaction Time (RT), Skin-Crossing Saccades, and Solution Rate As a Function of Group

\begin{tabular}{|c|c|c|c|c|c|c|c|}
\hline \multirow[b]{2}{*}{ Group } & \multirow[b]{2}{*}{$N$} & \multicolumn{2}{|c|}{ Number Dropped } & \multirow{2}{*}{$\begin{array}{c}\begin{array}{c}\text { Tracking } \\
\text { Task }\end{array} \\
\begin{array}{c}\text { RT } \\
(\mathrm{msec})\end{array}\end{array}$} & \multicolumn{2}{|c|}{ Skin-Crossing Saccades } & \multirow[b]{2}{*}{$\begin{array}{c}\text { Solution } \\
\text { Rate }\end{array}$} \\
\hline & & $\begin{array}{l}\text { Tracking } \\
\text { Failure }\end{array}$ & $\begin{array}{c}\text { Saw } \\
\text { Relationship }\end{array}$ & & $\begin{array}{c}\text { Free-Viewing } \\
\text { Period }^{\mathrm{a}}\end{array}$ & $\begin{array}{c}\text { Tracking } \\
\text { Task }^{\mathrm{b}}\end{array}$ & \\
\hline Embodied solution & 25 & 5 & 2 & $662^{*}$ & 0.3 & $6.7^{*}$ & $0.50^{*}$ \\
\hline Areas of interest & 25 & 6 & 1 & $643^{*}$ & 0.3 & $2.8^{*}$ & 0.33 \\
\hline Repeated skin crossing & 24 & 5 & 3 & $624^{*}$ & 0.4 & $6.5^{*}$ & 0.19 \\
\hline Tumor fixation & 25 & 7 & 0 & 494 & 0.3 & 0.4 & 0.22 \\
\hline
\end{tabular}

aSaccades/sec. bSaccades $/ 4 \mathrm{sec}$. *Statistical difference between an experimental condition and the control condition (tumor-fixation group) at the $p<.05$ level. 
with one item per location: upper left corner, center, upper right corner, center, lower right corner, center, lower left corner, center (see Figure 1B).

Participants in the areas-of-interest group visited the same locations during the tracking task as did participants in the embodiedsolution group, but the order in which tracking items appeared in this group deemphasized skin-crossing saccades. The order of locations at which tracking items appeared for the areas-of-interest group was: upper left corner, upper right corner, lower right corner, lower left corner, center, center, center, center (see Figure 1C).

Participants in the repeated-skin-crossing group ${ }^{4}$ performed a tracking task which emphasized skin-crossing saccades, all of which were between the same two locations. The order of tracking item locations for this group was: upper left corner, center, upper left corner, center, upper left corner, center, upper left corner, center (see Figure 1D).

Finally, participants in the tumor-fixation group performed a tracking task in which the eyes remained fixated in the center location throughout the entire 4-sec tracking task. All eight items on any given trial were presented sequentially in the center location, such that no skin-crossing saccades were required during the tracking task (see Figure 1E).

\section{Problem Solution and Experiment Completion}

In addition to performing the letter/digit-tracking task, participants also worked on solving the radiation problem. The participant was free at any time to stop the experiment and guess the problem's solution. A participant confident of having solved the problem pressed a game pad key with the left index finger. This action paused the current trial and brought up a display of the problem. An assistant placed tracing paper over the display and asked the participant to draw the solution on the paper. If the solution was correct (i.e., showed at least two lines from different outer locations crossing the skin to converge at the tumor), the experiment ended; if it was incorrect, the participant restarted the current trial and was free to guess again at any time. The experiment concluded whenever a participant gave a correct solution to the problem, or after $10 \mathrm{~min}$ at the task (i.e., 20 tracking sequences), whichever happened first.

After the experiment, participants completed a short questionnaire which asked whether participants saw a relationship between the radiation problem and the letter/digit tracking task.

\section{RESULTS}

\section{Tracking Task}

We evaluated the performance of each participant on the letter/digit tracking task. Table 1 shows the mean digit identification RT for each of the experimental groups. The main effect of group on RT was significant in a one-way ANOVA $\left[F(3,67)=5.77, M S_{\mathrm{e}}=17,716, p=.001\right]$. A Tukey planned comparison confirmed that RTs for the tumor-fixation group were significantly faster than the RTs in the embodied-solution group (mean difference $=$ 169 , standard error $[S E]=46, p=.003)$, the areas-ofinterest group (mean difference $=149, S E=44, p=$ .007 ), and the repeated-skin-crossing group (mean difference $=130, S E=46, p=.030$ ), whereas the RTs for the embodied-solution group, areas-of-interest group, and repeated-skin-crossing group were not significantly different (all $p \mathrm{~s}>.8$ ). This RT advantage was to be expected, given that participants in the tumor-fixation group did not have to move their eyes in order to foveate the stimuli and therefore had the advantage of viewing each stimulus at fixation for its entire 500-msec presentation time.

In order to determine the effectiveness of the tracking task in eliciting the desired eye movement patterns, we counted the number of skin-crossing saccades participants made during the 4-sec tracking task. Figure 2A shows the average number of skin-crossing saccades per 4-sec tracking interval that participants in each of the four groups made. The main effect of group was significant in a one-way ANOVA $\left[F(3,67)=123.46, M S_{\mathrm{e}}=82.97\right.$, $p<.001]$. Post hoc tests confirmed that the difference between the number of skin-crossing saccades in the embodied-solution and repeated-skin-crossing groups was not significant (Tukey test mean difference $=0.23, S E=$ $0.40, p=.942)$, whereas all other pairwise differences were significant (all $p \mathrm{~s}<.001$ ). These results confirmed that our manipulations were effective in creating differences between groups in the number of skin-crossing saccades (with the exception of the embodied-solution and repeated-skin-crossing groups, for which the numbers of skin-crossing saccades were intentionally matched).

We also examined whether there were any differences between the rates of skin-crossing saccades between groups during the 26-sec free-viewing period (i.e., excluding the eye movement data collected during the tracking task). These data are shown in Figure 2B. As can be seen from this figure, during the free-viewing period participants tended to make skin-crossing saccades equally often regardless of group. This observation was confirmed by a one-way ANOVA $\left[F(3,67)=1.08, M S_{\mathrm{e}}=0.03, p=.363\right]$.

\section{Problem-Solving Task}

Our primary question of interest was whether the eye movements that participants made during the letter/digit tracking task influenced their chances of successfully solving the radiation problem. The rates of problem-solving success by the end of the 10-min period for each of the four groups are shown in Table 1. Figure 3 shows the proportion of participants in each group to successfully solve the problem after each trial. A Peto-Peto-Prentice survival analysis test (see Cleves, Gould, \& Gutierrez, 2002, for details) comparing the solution rates for all four groups across the twenty $30-$ sec intervals suggested that these rates were marginally different between the four groups $\left[\chi^{2}(3, N=70)=7.48, p=.058\right]$. Planned pairwise comparisons between groups showed that this effect was driven by a significant difference in solution rates between the embodied-solution and the tumor-fixation groups $\left[\chi^{2}(1, N=36)=5.10, p=.024\right]$ and a significant difference in solution rates between the embodied-solution and repeated-skin-crossing groups $\left[\chi^{2}(1, N=34)=3.87\right.$, $p=.049]$; no other pairwise comparisons were statistically significant (all $p \mathrm{~s}>$.2). Furthermore, a comparison of solution rates for the embodied-solution group versus the areas-of-interest, tumor-fixation, and repeated-skincrossing groups combined was also significant $\left[\chi^{2}(1, N=\right.$ $70)=5.95, p<.015]$.

In addition to investigating the possible links between eye movement patterns and problem-solving success rates, we also wished to determine whether these potential links were implicit or explicit. A check of the completed posttest questionnaires suggested that few participants suspected a relationship between the tracking task and the insight problem. In fact, many believed the tracking task was a 

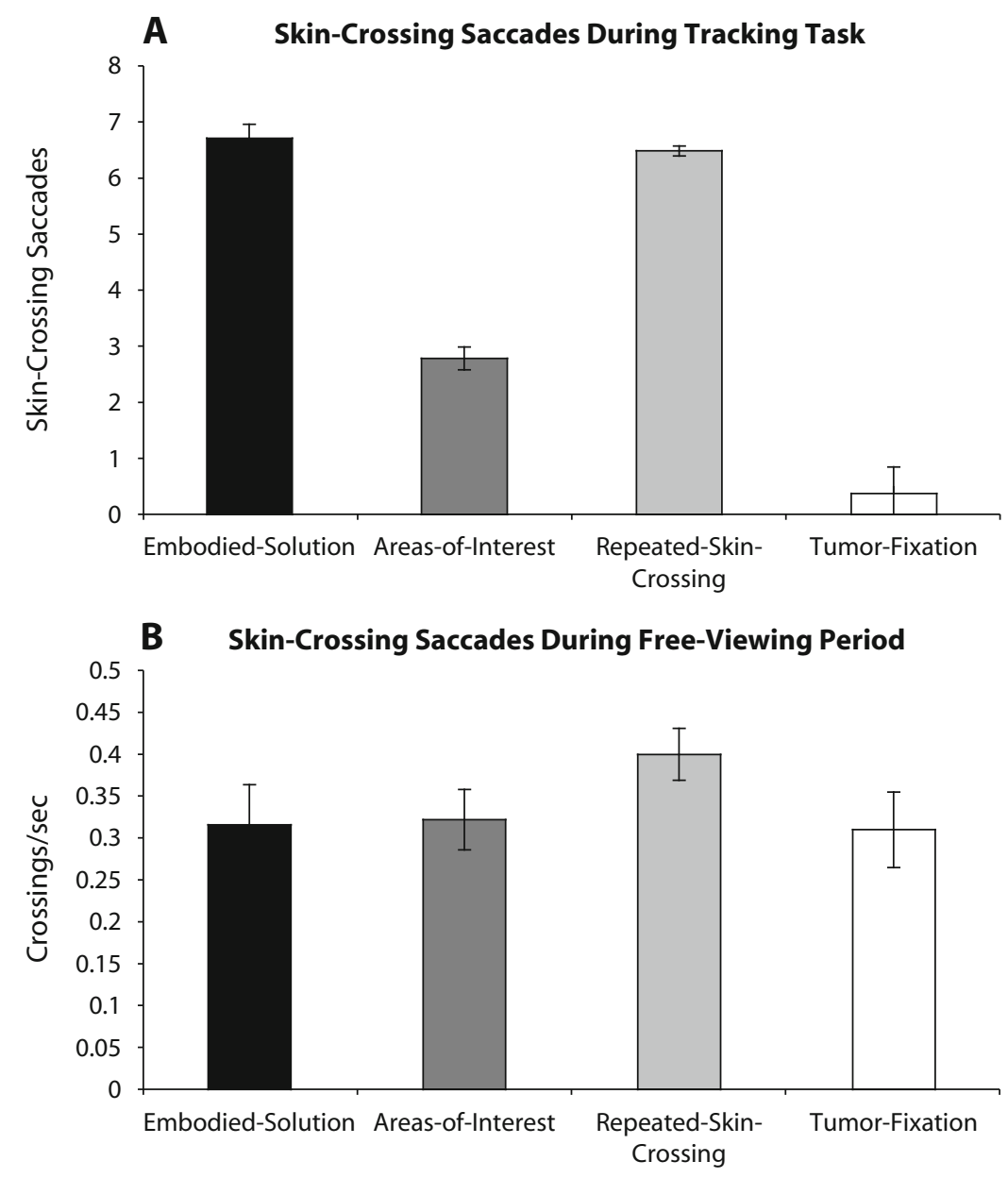

Figure 2. Mean number of skin-crossing saccades for the embodied-solution group, areas-of-interest group, repeated-skin-crossing group, and tumor-fixation group. Panel A shows the mean number of total skin-crossing saccades during the tracking task period only. Panel B shows the mean number of skin-crossing saccades per second recorded during the free-viewing periods only (i.e., excluding eye movements made during the digit tracking task).

purposeful distraction from the radiation problem. Only 6 participants in total suspected a relationship between the tracking and problem-solving tasks, and their data were not included in the problem-solving analyses.

\section{DISCUSSION}

Our results show that participants in the embodiedsolution group were more likely to solve the radiation problem than were participants in all of the other groups. This result is consistent with the hypothesis that eye movement patterns can influence thought, at least in spatial reasoning tasks such as Duncker's (1945) radiation problem. To the extent that our postexperimental questionnaire accurately captured how participants perceived the relationship between the radiation problem and the tracking task, our results suggest that this link between eye movements and cognition was implicit. Guided eye movements in this experiment were not viewed by participants as overt "hints" at the problem's solution, but rather as distract- ing and interfering with the Duncker task. Taken together, these results provide evidence for an implicit link between eye movement patterns and spatial cognition; furthermore, they also suggest the nature of the relationship between eye movements and problem solving. Participants were most successful in solving the radiation problem when their eyes were guided in skin-crossing saccades over multiple locations; in contrast, directing attention to these locations without the skin-crossing saccades, or repeatedly guiding the eyes across the skin in the same location, did not mean that participants were more likely to solve the problem than they were when their eyes simply fixated the tumor. In order to be most effective in influencing problem-solving success, guided eye movements needed to specifically embody a solution to the problem.

Interestingly, although the results of the analysis of eye movements during the tracking task period show that our manipulation was effective in establishing differences between the patterns of eye movements across groups, the analysis of eye movements during the free- 


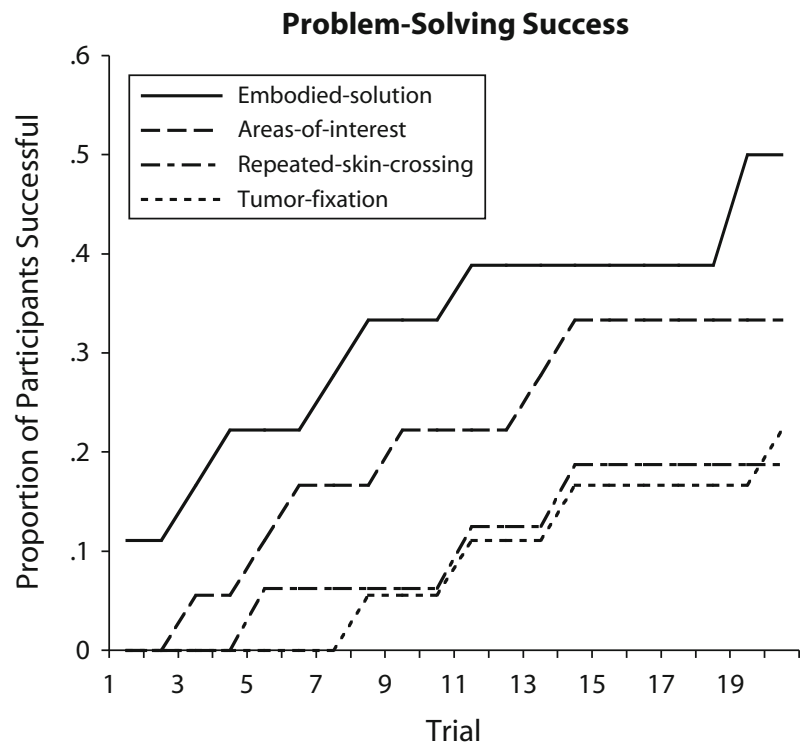

Figure 3. Proportion of participants in each group to successfully solve Duncker's (1945) radiation problem after each trial.

viewing period suggests that these differences were not carried over when participants were free to look anywhere onscreen. When participants were not engaged in the tracking task, they tended to make the same number of skin-crossing saccades, regardless of how their eyes moved during the tracking task. In other words, a relatively brief manipulation-guiding the eyes for $4 \mathrm{sec}$ out of every $30 \mathrm{sec}$ - that did not influence oculomotor behavior for the remainder of a trial had a significant impact on problem-solving success. That there was no contamination of the tracking task on eye movement behavior during the free-viewing periods lends additional support to the argument that the effects of eye movement patterns on spatial cognition are implicit in nature; had participants been aware of the relationship between the tracking task and the radiation problem, we might have expected them to make eye movements similar to those induced by the tracking task during the free-viewing period.

In sum, our results provide strong support to Grant and Spivey's (2003) proposal that a specific pattern of eye movements-skin-crossing saccades made over different regions of the radiation problem diagram-can serve as an embodied physical mechanism that initiates a perceptual simulation, guiding participants toward the insight that multiple lasers must be fired from different points outside the diagram. These studies join a growing body of literature on embodied cognition showing that cognitive work can be offloaded onto the environment and, moreover, that cognitive processes can actually arise from, and be influenced by, the manners in which our bodies interact with our immediate environment (see Iverson \& GoldinMeadow, 1998; Krauss, 1998; Wilson, 2002).

We believe that eye movement trajectories can serve as implicit "thought" guides in spatial reasoning tasks. By directing eye movements for only a small portion of participants' problem-viewing time, we were able to sub- stantially affect their chances of problem-solving success. Although additional studies are necessary to determine how powerful this link between eye movements and cognition is, it is now clear that not only do eye movements reflect what we are thinking, they can also influence how we think.

\section{AUTHOR NOTE}

The authors thank Larry Barsalou, Jonathan Fugelsang, Bobbie Spellman, and Michael Spivey for their comments on the research, and Shanqing Yin, Kristin Stewart, Mariann Chelampath, and Li-Eng Chen for their assistance with data collection. This research was supported by an NSF Graduate Research Fellowship to L.E.T. and an NSF grant to A.L., Award 0527361. Correspondence concerning this article should be addressed to L. E. Thomas, Department of Psychology, University of Illinois at Urbana-Champaign, 603 E. Daniel St., Champaign, IL 61820 (e-mail: lethomas@cyrus.psych.uiuc.edu).

\section{REFERENCES}

Barsalou, L. W. (1999). Perceptual symbol systems. Behavioral \& Brain Sciences, 22, 577-600.

Cleves, M. A., Gould, W. W., \& Gutierrez, R. G. (2002). An introduction to survival analysis using Stata. College Station, TX: Stata Press.

Duncker, K. (1945). On problem solving. Psychological Monographs, 58(5, Whole No. 270).

Grant, E. R., \& Spivey, M. J. (2003). Eye movements and problem solving: Guiding attention guides thought. Psychological Science, 14, 462-466.

IvERSON, J. M., \& Goldin-Meadow, S. (1998). Why people gesture when they speak. Nature, 396, 228.

Just, M. A., \& CARPENTER, P. A. (1985). Cognitive coordinate systems: Accounts of mental rotation and individual differences in spatial ability. Psychological Review, 92, 137-172.

Knoblich, G., Ohlsson, S., \& Raney, G. E. (2001). An eye movement study of insight problem solving. Memory \& Cognition, 29, 1000-1009.

KRAUSS, R. M. (1998). Why do we gesture when we speak? Current Directions in Psychological Science, 7, 54-60.

LENHART, R. E. (1983). Conjugate lateral eye movements and problem solving ability; or, where to look for the right answer. Psychophysiology, 20, 456.

Metcalfe, J., \& Wiebe, D. (1987). Intuition in insight and noninsight problem solving. Memory \& Cognition, 15, 238-246.

Weisberg, R. W., \& AlbA, J. W. (1981). An examination of the alleged role of "fixation" in the solution of several "insight" problems. Journal of Experimental Psychology: General, 110, 169-192.

WILSON, M. (2002). Six views of embodied cognition. P sychonomic Bulletin \& Review, 9, 625-636.

\section{NOTES}

1. Insight problems are characterized by the fact that solutions that seem most obvious to naive problem solvers do not work, that problem solvers working on them cannot accurately track their own performance, and that problem solvers must often overcome an impasse in their reasoning in order to infer the problem's solution (Metcalfe \& Wiebe, 1987; Weisberg \& Alba, 1981).

2. Although participants in the repeated-skin-crossing group were run separately from participants in the other groups, they were recruited from the same pool and given identical incentives to participate.

3. An EyeLink 1000 video-based eyetracker (SR Research Ltd.) recorded participants' eye movements in the repeated-skin-crossing group. This system had comparable temporal, spatial, and pupil-size resolution and classified saccades in the same manner as the EyeLink II.

4. We thank Lawrence W. Barsalou for suggesting this manipulation.

(Manuscript received April 14, 2006; revision accepted for publication October 2, 2006.) 